\title{
On mathematical theory of selection: continuous time population dynamics
}

\author{
Georgiy P. Karev
}

Received: 28 December 2007 / Revised: 27 January 2009 / Published online: 13 March 2009

(C) The Author(s) 2009. This article is published with open access at Springerlink.com

\begin{abstract}
Mathematical theory of selection is developed within the frameworks of general models of inhomogeneous populations with continuous time. Methods that allow us to study the distribution dynamics under natural selection and to construct explicit solutions of the models are developed. All statistical characteristics of interest, such as the mean values of the fitness or any trait can be computed effectively, and the results depend in a crucial way on the initial distribution. The developed theory provides an effective method for solving selection systems; it reduces the initial complex model to a special system of ordinary differential equations (the escort system). Applications of the method to the Price equations are given; the solutions of some particular inhomogeneous Malthusian, Ricker and logistic-like models used but not solved in the literature are derived in explicit form.
\end{abstract}

Keywords Selection system - Dynamics of distribution - The Price equation . Inhomogeneous logistic model $\cdot$ Replicator equation

Mathematics Subject Classification (2000) $\quad 37$ N25 $\cdot 92$ A 30

\section{Background}

The problem of construction of a formal general theory of selection was clearly formulated in (Price 1970, 1995). The Haldane principle Haldane (1990), the Fisher Fundamental theorem of natural selection (FTNS) Fisher (1999), the covariance equation (Li 1967; Robertson 1968) and the Price equation Price (1970, 1972) were the first

G. P. Karev $(\varangle)$

Lockheed Martin MSD, National Institutes of Health, Bldg. 38A, Rm. 5N511N,

8600 Rockville Pike, Bethesda, MD 20894, USA

e-mail: karev@ncbi.nlm.nih.gov 
outstanding contributions to the future theory. The covariance equation and the FTNS are particular cases of the Price equation (see Page and Nowak 2002).

Another general approach to the formal theory of selection, models with inheritance, was developed in the 1970 s to the 1980 s in the works of S. Semenov, V. Okhonin, A. Gorban, et al. (see Gorban 2007 for references). The approach was based on an abstract version of the so-called replicator equation Hofbauer and Sigmund (1998), Schuster and Sigmund (1983). The Haldane principle and the Gause competition principle Gause (1934) were proven and explored as mathematical assertions within the frameworks of the developed formalism. Recently Grafen (2006) also derived the Price equations and the FTNS in a very general form. Both theories are interesting and promising but, perhaps, too abstract for most biological applications.

In this paper we develop an approach to general selection systems that can be applied directly to many mathematical models. We study the evolution of system distributions and obtain the main results in the explicit form. We show that knowing the initial distribution allows us to predict the system dynamics indefinitely and, in particular, to resolve the problem of "dynamical insufficiency" of the Price equations. The developed methods are applicable to a wide class of inhomogeneous population models. We show that the initial complex model can be reduced to the "escort system" of ODEs for auxiliary variables and then solved effectively.

The paper is organized as follows. The master model for selection systems is introduced in Sect. 2. Selection models with self-regulated fitness are explored in Sect. 3, which also contains the main mathematical results, Theorem 1 and its Corollary. Section 4.1 contains applications of the theory to the Price equation; the algorithm for solving the selection systems and corresponding replicator equations is described in Sect. 4.2; evolution of particular distributions of biological interest governed by selection over a single or several traits is studied in Sect. 4.3. Explicit solutions of some inhomogeneous Malthusian and logistic-like models used in literature are derived in Sect. 5

\section{Master model for selection systems}

Let us consider a general model of inhomogeneous population, in which every individual is characterized by a vector-parameter $\left(a_{1}, \ldots, a_{n}\right)=\mathbf{a}$ that takes values from set $A$. The parameter a specifies an individual's inherited invariant properties and may vary from one individual to another, such that the population is non-uniform. Any changes of the parameter distribution with time are caused only by variation of the population structure.

The set of all individuals with a given value of the vector-parameter $\mathbf{a}$ in the population is called a-clone. Let $l(t, \mathbf{a})$ be the density of the population at the moment $t$; informally, $l(t, \mathbf{a})$ is the number of individuals in the a-clone. Let us denote $F(t, \mathbf{a})$ the per capita reproduction rate (Malthusian fitness) of the a-clone at the moment $t$. We suppose that the reproduction rate of every a-clone does not depend on other clones, but can depend on a and on the "environment" at $t$ moment, which, in turn, may depend on the total population size $N(t)$ and other population characteristics. These quantities evolve with time, however, for a given set of initial conditions have specific values at each point in time for each value of $\mathbf{a}$. The exact form of the reproduction rate will be specified in Sect. 3 . 
If we assume the overlapping generations and smoothness of $l(t, \mathbf{a})$ in $t$ for each $\mathbf{a} \in A$, then the population dynamics can be described by the following master model:

$$
\begin{aligned}
d l(t, \mathbf{a}) / d t & =l(t, \mathbf{a}) F(t, \mathbf{a}), \\
N(t) & =\int_{A} l(t, \mathbf{a}) d \mathbf{a}, \\
P(t, \mathbf{a}) & =l(t, \mathbf{a}) / N(t)
\end{aligned}
$$

where $P(t, \mathbf{a})$ is the probability density function (pdf) at $t$ instant. The initial pdf $P(0, \mathbf{a})$ and the initial population size $N(0)$ are assumed to be given. Equations (2.1) comprise the formal (after G. Price) selection system with continuous time.

In what follows any characteristic that is inherent to the individual, is fixed at the very beginning of the individual life and does not change with time we refer to as a trait. The selection system describes a closed population of individuals each of which is characterized by a set of qualitative traits; the values of these traits determine the reproduction rate of the individual. It is supposed that the mean values of the traits are the only information that is known about the entire population. The dynamics of the joint distribution of these traits depending on the initial distribution and on correlations between the traits is the main problem of interest.

Hereinafter we use the notation $E^{t}[f]=\int_{A} f(\mathbf{a}) P_{t}(\mathbf{a}) d \mathbf{a}$. It is known (and can be easily proven) that the population size $N(t)$ satisfies the equation

$$
d N / d t=N E^{t}[F]
$$

and the pdf $P(t, \mathbf{a})$ solves the replicator equation

$$
d P(t, \mathbf{a}) / d t=P(t, \mathbf{a})\left(F(t, \mathbf{a})-E^{t}[F]\right) .
$$

The problems (2.1) and (2.2), (2.3) are equivalent.

Taking into account that any smooth function $F(t, \mathbf{a})$ can be well approximated by a finite sum of the form $\sum_{i} f_{i}(t) \varphi_{i}(\mathbf{a})$, we will suppose that the reproduction rate is of the form

$$
F(t, \mathbf{a})=\sum_{i=1}^{n} f_{i}(t) \varphi_{i}(\mathbf{a}) .
$$

Biologically it means that we consider the individual fitness that depends on a given finite set of traits, or "predictors" (see Frank 1997; Goodnight 1992; Lande and Arnold 1983, etc.). The function $\varphi_{i}$ (a) in (2.4) may describe quantitative contribution of a particular $i$ th trait to the total fitness and then $f_{i}(t)$ are corresponding coefficients of multiple regression. In more sophisticated models, the functions $f_{i}(t)$ describe relative importance of the trait contributions depending on the environment, population size, etc. 
The functions $f_{i}(t)$ can be known explicitly at any time moment but it is not the case for most realistic models, which accounts for self-limitations of the population growth. For example, even the simple inhomogeneous logistic model with $F(t, \mathbf{a})=\varphi(\mathbf{a})(1-N(t) / B)$ where constant $B$ is the carrying capacity, does not satisfy this condition because the current population size $N_{t}$ is unknown a priori. Therefore we should investigate a class of models (2.1), (2.4) where $f_{i}$ are the functions of the total population size and other population characteristics (see Sect. 3), which are not given and should be computed at every time moment. We show in this paper that this non-trivial problem can be solved effectively.

\section{Self- regulated selection system}

Suppose that the individual reproduction rate can depend on some integral characteristics of the system; we account for extensive characteristics, which depend on the total size of the system (as in most population models) and intensive characteristics, which do not depend on the total size but only on the population frequencies (as in most genetic models). We consider the intensive characteristics in the form

$$
H(t)=\int_{A} h(\mathbf{a}) P(t, \mathbf{a}) d \mathbf{a}=E^{t}[h]
$$

and the extensive characteristics in the form

$$
G(t)=\int_{A} g(\mathbf{a}) l(t, \mathbf{a}) d \mathbf{a}=N(t) E^{t}[g],
$$

where $g, h$ are appropriate weight functions. Both expressions (3.1) and (3.2) are known also as "generalized variables" or "regulating functionals"; we will refer to them as "regulators" for brevity. The total system size $N(t)$ is also a regulator (3.2) at $g(\mathbf{a}) \equiv 1$ and is of a special importance.

Suppose that the fitness of every individual is determined by a given set of traits; suppose also that the reproduction rate of every a-clone may depend on the size and frequency of other clones only through the regulators. Then we obtain the following general version of the master model:

$$
\begin{aligned}
d l(t, \mathbf{a}) / d t & =l(t, \mathbf{a}) F(t, \mathbf{a}), \\
F(t, \mathbf{a}) & =\sum_{i=1}^{n} u_{i}\left(t, G_{i}\right) \varphi_{i}(\mathbf{a})+\sum_{k=1}^{m} v_{k}\left(t, H_{k}\right) \psi_{k}(\mathbf{a}), \\
P(t, \mathbf{a}) & =l(t, \mathbf{a}) / N(t)
\end{aligned}
$$

where $G_{i}, H_{k}$ are the regulators, $u_{i}, v_{k}$ are given functions. The initial pdf $P(0, \mathbf{a})$ and the initial population size $N(0)$ are supposed to be given. In model (3.3) with self-regulated fitness the regulators and hence the reproduction rate $F(t, \mathbf{a})$ are not 
given as explicit functions of time but should be computed employing the current pdf $P(t, \mathbf{a})$ at each time moment.

Let us now formulate the basic theorem for model (3.1)-(3.3). Let $\Phi(r ; \boldsymbol{\lambda}, \boldsymbol{\delta})$ be the generating functional

$$
\Phi(r ; \boldsymbol{\lambda}, \boldsymbol{\delta})=\int_{A} r(\mathbf{a}) \exp \left(\sum_{i=1}^{n} \lambda_{i} \varphi_{i}(\mathbf{a})+\sum_{k=1}^{m} \delta_{k} \psi_{k}(\mathbf{a})\right) P(0, \mathbf{a}) d \mathbf{a}
$$

where $\lambda=\left(\lambda_{1}, \ldots \lambda_{n}\right), \delta=\left(\delta_{1}, \ldots \delta_{m}\right)$ and $r(\mathbf{a})$ is a measurable function on $A$.

Define auxiliary variables as a solution of the escort system of differential equations:

$$
\begin{array}{ll}
d q_{i} / d t=u_{i}\left(t, G_{i}^{*}(t)\right), & q_{i}(0)=0, \quad i=1, \ldots n, \\
d s_{k} / d t=v_{k}\left(t, H_{k}^{*}(t)\right), & s_{k}(0)=0, \quad k=1, \ldots m
\end{array}
$$

where

$$
\begin{aligned}
& N^{*}(t)=N(0) \Phi(1 ; \mathbf{q}(t), \mathbf{s}(t)) \\
& G_{i}^{*}(t)=N(0) \Phi\left(g_{i} ; \mathbf{q}(t), \mathbf{s}(t)\right) \\
& H_{i}^{*}(t)=\Phi\left(h_{i} ; \mathbf{q}(t), \mathbf{s}(t)\right) / \Phi(1 ; \mathbf{q}(t), \mathbf{s}(t))
\end{aligned}
$$

Let us denote

$$
K_{t}(\mathbf{a})=\exp \left(\sum_{i=1}^{n} q_{i}(t) \varphi_{i}(\mathbf{a})+\sum_{k=1}^{m} s_{k}(t) \psi_{k}(\mathbf{a})\right)
$$

The function $K_{t}(\mathbf{a})$ is the reproduction coefficient for the time interval $[0, t)$, or $t$ fitness for short (see formula (3.8) below). Notice that $E^{0}\left[K_{t}\right]=\Phi(1 ; \mathbf{q}(t), \mathbf{s}(t))$.

The following main theorem reduces model (3.3) to a Cauchy problem for the escort system.

Theorem 1 Let $0<T \leq \infty$ be the maximal value of t such that Cauchy problem(3.5), (3.6) has a unique global solution $\{\mathbf{q}(t), \mathbf{s}(t)\}$ at $t \in[0, T)$. Then the functions

$$
\begin{aligned}
l(t, \mathbf{a}) & =l(0, \mathbf{a}) K_{t}(\mathbf{a}) \\
N(t) & =N(0) \Phi(1 ; \mathbf{q}(t), \mathbf{s}(t)) \\
G_{i}(t) & =N(0) \Phi\left(g_{i} ; \mathbf{q}(t), \mathbf{s}(t)\right) \\
H_{i}(t) & =\Phi\left(h_{i} ; \mathbf{q}(t), \mathbf{s}(t)\right) / \Phi(1 ; \mathbf{q}(t), \mathbf{s}(t))
\end{aligned}
$$

satisfy system (3.1)-(3.3) at $t \in[0, T)$.

Conversely, if $l(t, \mathbf{a})$ solves system (3.3) at $t \in[0, T)$ so that $N(t)=\int_{A} l(t, \mathbf{a}) d \mathbf{a}$, $H_{k}(t)=\int_{A} h_{k}(\mathbf{a}) l(t, \mathbf{a}) d \mathbf{a} / N(t), G_{i}(t)=\int_{A} g_{i}(\mathbf{a}) l(t, \mathbf{a}) d \mathbf{a}$, then Cauchy problem (3.5), (3.6) has a global solution $\{\mathbf{q}(t), \mathbf{s}(t)\}$ at $t \in[0, T)$ and the functions $l(t, \mathbf{a}), N(t), G_{i}(t), H_{k}(t)$ can be written in the form (3.8)-(3.11).

The proof of Theorem 1 is given in Appendix. 


\section{Corollary 1 The current system distribution}

$$
P(t, \mathbf{a})=P(0, \mathbf{a}) K_{t}(\mathbf{a}) / E^{0}\left[K_{t}\right] .
$$

The last formula is the central result of the theory, which gives the solution of replicator equation (2.3). Theorem 1 gives an effective algorithm for investigation of the selection systems (see Sect. 6). It allows us to define and compute the total population size and the values of all regulators at any time moment. After that, the reproduction rate $F(t, \mathbf{a})$ in model (3.3) can be considered as a known function of time. In this particular case the theory of selection systems is rather simple (see, e.g., Karev 2005b) and all its results can now be applied to self-regulated model (3.3).

\section{Dynamics of the system distribution}

\subsection{The Haldane' principle and the Price equation}

It is known that any stationary distribution of a system with inheritance, and of system (2.1) in particular should be concentrated in the set of points of global maximum of the average reproduction rate on the support of initial distribution; this maximal value must be equal to 0 , otherwise the limit distributions cannot exist. This version of the Haldane extreme principle was established in Gorban (1984, 2007), Semevsky and Semenov (1982). Similar "selection principle" was proven in Perthame (2007), Sect. 2.1, 2.2 for logistic-like models. The Haldane principle predicts the behavior of selection systems "at infinity" if the limit distribution exists and is stable. Note that the last condition is not necessarily fulfilled even for the commonly used models; the simplest examples are inhomogeneous Malthusian and some logistic models, see Sect. 5.

On the other end of the time scale, the Price equation describes the instant change of the mean value of individual characters for any selection system. Within the framework of model (2.1) a character, which may depend on time, can be considered a random variable $z(t, \mathbf{a})$ (formally defined on the probabilistic space $\{A, \mathbf{A}, P(t, \mathbf{a})\}$ where $\mathbf{A}$ is a $\sigma$-algebra of Borel subsets of $A$ ). Then the Price equation (Page and Nowak 2002; Price 1970, 1972, 1995; Rice 2006) states that

$$
d E^{t}\left[z_{t}\right] / d t=\operatorname{Cov}^{t}\left[F, z_{t}\right]+E^{t}\left[d z_{t} / d t\right]
$$

It is well known (Barton and Turelli 1987; Frank 1997; Lewontin 1974, etc.) that the Price equation is dynamically insufficient, i.e., it cannot be used alone as a propagator of the dynamics of the model forward in time. We can write the Price equation in, perhaps, intuitively more clear integral form that shows the connection between the reproduction coefficient and the selection differential $\Delta_{t} z=E^{t}\left[z_{t}\right]-E^{0}\left[z_{0}\right]$, which is an important characteristic of selection.

Let us define the reproduction coefficient for the time interval $[s, s+t)$ as $k_{s}^{s+t}(\mathbf{a})=$ $\exp \left(\int_{s}^{s+t} F(u, \mathbf{a}) d u\right)$. Denote $k_{t}(\mathbf{a})=k_{0}^{t}(\mathbf{a})$ for brevity; remark that $k_{t}(\mathbf{a})=K_{t}(\mathbf{a})$ 
for model (3.3). Then

$$
E^{t+s}\left[z_{t+s}\right]-E^{t}\left[z_{t}\right]=\operatorname{Cov}^{t}\left[z_{t+s}, k_{t}^{t+s}\right] / E^{t}\left[k_{t}^{t+s}\right]+E^{t}\left[z_{t+s}-z_{t}\right] .
$$

In particular,

$$
E^{s}\left[z_{s}\right]=\operatorname{Cov}^{0}\left[z_{s}, k_{s}\right] / E^{0}\left[k_{s}\right]+E^{0}\left[z_{s}\right] .
$$

Notice that this version of the Price equation is quite similar to the one for discretetime models Karev (2008), as opposed to the differential version (4.1). Taking into consideration that $K_{t}^{t+s}(\mathbf{a}) \approx 1+s F(\mathbf{a})$ we can see that the integral relation (4.2) turns to differential Price' equation (4.1) as $s \rightarrow 0$.

It is worth emphasizing that there exists only one reason for "dynamical insufficiency" of the Price equation: this equation is a mathematical identity. Within the frameworks of master model (2.1) the theory developed above helps resolve the problem of dynamical insufficiency of the Price equation and its particular cases, the covariance equation and the equation of the FTNS. Formula (3.12) allows us to compute the mean value of any character in any time moment if the initial pdf is known; in this sense, the following proposition gives the "solution" of the Price equation.

Proposition 1 Given the initial distribution, the solution of the Price equation is given by the formulas

i) $E^{t}\left[z_{t}\right]=E^{0}\left[z_{t} k_{t}\right] / E^{0}\left[k_{t}\right]$ for model $(2.1)$;

ii) $E^{t}\left[z_{t}\right]=\Phi\left(z_{t} ; \mathbf{q}(t), \mathbf{s}(t)\right) / \Phi(1 ; \mathbf{q}(t), \mathbf{s}(t))$ for self-regulated model (3.3) where the auxiliary variables $\mathbf{q}(t), \mathbf{s}(t)$ solve the escort $O D E$ system (3.5).

\subsection{How to study selection systems and the dynamics of their distribution?}

Not only asymptotic behavior, but also the current dynamics of the population distribution during protracted but finite time intervals are of interest and, perhaps, of primary importance for applications.

The developed theory yields an effective algorithm to analyze selection systems, which reduces complex self-regulated selection system to the escort system of nonautonomic ODEs. Let us summarize the main steps of the algorithm.

We study a model of selection system in the form (3.3). In applications, the functions $\varphi_{i}(\mathbf{a}), \psi_{k}(\mathbf{a})$ can be interpreted as traits that characterize an individual; $u_{i}\left(t, G_{i}\right)$ and $v_{k}\left(t, H_{k}\right)$ describe the contribution of the corresponding traits to the individual fitness at moment $t$. The following steps should be performed for solving a particular selection system:

1) composing generating functional (3.4);

2) solving escort system of ODE (3.5).

3) After that, the solution to the selection system $l(t, \mathbf{a})$, the population size $N(t)$, and the values of regulators at $t$ moment are given by formulas (3.7)-(3.11);

4) the current system distribution is given by formula (3.12), which allows us to compute all statistical characteristics of interest for a self-regulated selection system. 
Generating functional (3.4) is the main tool of the suggested approach; it may be difficult to compute it in general case. Remark, that we in fact do not need to know this functional for arbitrary function $r$ but only for the functions $g_{k}, k=1, \ldots, m$, and $h_{i}, i=$ $1, \ldots, n$, see (4.1) and (4.2). So, instead of generating functional (3.4) we can use the moment generating function of the initial joint distribution of the set of random variables $\left\{\varphi_{i}, g_{i}, \psi_{k}, h_{k}\right\}$,

$$
\begin{aligned}
M_{0}(\boldsymbol{\lambda}, \boldsymbol{\delta}, \boldsymbol{\gamma}, \boldsymbol{\eta})= & \int_{A} \exp \left(\sum_{i=1}^{n}\left(\lambda_{i} \varphi_{i}(\mathbf{a})+\gamma_{i} g_{i}(\mathbf{a})\right)\right. \\
& \left.+\sum_{k=1}^{m}\left(\delta_{k} \psi_{k}(\mathbf{a})+\eta_{k} h_{k}(\mathbf{a})\right)\right) P(0, \mathbf{a}) d \mathbf{a} .
\end{aligned}
$$

Indeed,

$$
\begin{aligned}
\Phi\left(g_{i} ; \mathbf{q}(t), \mathbf{s}(t)\right) & =\int_{A} g_{i}(\mathbf{a}) \exp \left(\sum_{i=1}^{n} q_{i}(t) \varphi_{i}(\mathbf{a})+\sum_{k=1}^{m} s_{k}(t) \psi_{k}(\mathbf{a})\right) P(0, \mathbf{a}) d \mathbf{a} \\
& =\left.\frac{\partial}{\partial \gamma_{i}} M_{0}(\mathbf{q}(t), \mathbf{s}(t), \boldsymbol{\gamma}, \boldsymbol{\eta})\right|_{\boldsymbol{\gamma}=\boldsymbol{\eta}=0}, \\
\Phi\left(h_{k} ; \mathbf{q}(t), \mathbf{s}(t)\right) & =\left.\frac{\partial}{\partial \nu_{k}} M_{0}(\mathbf{q}(t), \mathbf{s}(t), \boldsymbol{\gamma}, \boldsymbol{\eta})\right|_{\boldsymbol{\gamma}=\boldsymbol{\eta}=0},
\end{aligned}
$$

The general method is simplified in an important case of the reproduction rate $F(t, \mathbf{a})=$ $\sum_{i=1}^{n} f_{i}\left(t, S_{i}\right) \phi_{i}(\mathbf{a})$ with regulators $S$ of the forms $N(t), E^{t}\left[\phi_{i}\right], N(t) E^{t}\left[\phi_{i}\right]$ only. In this case we can use the moment generating function of the joint initial distribution of the variables $\left\{\phi_{i}\right\}$ only, $M_{0}(\lambda)=E^{0}\left[\exp \left(\sum_{i=1}^{n} \lambda_{i} \phi_{i}\right)\right]$, instead of general mgf (4.4). The escort system reads

$$
d q_{i} / d t=f_{i}\left(t, S_{i}(t)\right), \quad q_{i}(0)=0, i=1, \ldots n
$$

where $S_{i}(t)$ (having the form $N(t), E^{t}\left[\phi_{i}\right], N(t) E^{t}\left[\phi_{i}\right]$ ) are defined by the formulas

$$
\begin{aligned}
N(t) & =N(0) M_{0}(\mathbf{q}(t)), \\
E^{t}\left[\phi_{k}\right] & =\partial_{k} \ln M_{0}(\mathbf{q}(t)) .
\end{aligned}
$$

Here we denoted $\partial_{k} M_{0}(\lambda)=\partial M_{0}(\lambda) / \partial \lambda_{k}$ for brevity. This simplified version of the algorithm works for many models, see Sect. 5. It is important that the moment generating functions are known for most discrete and continuous distributions used in biological applications.

\subsection{Dynamics of particular distributions}

Let us formulate the following assertions that capture the dynamics of the system distribution for some probability distributions of biological interest. A selection system, 
whose evolution is governed by selection over a single trait, $\varphi(\mathbf{a})$, is the simplest but widely spread and important case. The reproduction rate may depend on regulators (3.1), (3.2); for simplicity, suppose that it depends only on extensive regulators like the total population size. Then the system is of the form

$$
\begin{aligned}
d l(t, \mathbf{a}) / d t & =l(t, \mathbf{a}) F(t, \mathbf{a}), \\
F(t, \mathbf{a}) & =u_{0}\left(t, G_{0}\right)+u_{1}\left(t, G_{1}\right) \varphi(\mathbf{a}), \\
G_{i}(t) & =\int_{A} g_{i}(\mathbf{a}) l(t, \mathbf{a}) d \mathbf{a} .
\end{aligned}
$$

The regulating functional for this system

$$
\Phi\left(r ; \lambda_{0}, \lambda_{1}\right)=\exp \left(\lambda_{0}\right) \int_{A} r(\mathbf{a}) \exp \left(\lambda_{1} \varphi(\mathbf{a})\right) P(0, \mathbf{a}) d \mathbf{a} .
$$

Define the auxiliary variables $q_{0}(t), q_{1}(t)$ by the escort system:

$$
\begin{aligned}
d q_{0} / d t & =u_{0}\left(t, N(0) \Phi\left(g_{0} ; q_{0}, q_{1}\right)\right), \\
d q_{1} / d t & =u_{1}\left(t, N(0) \Phi\left(g_{1} ; q_{0}, q_{1}\right)\right) \\
q_{i}(0) & =0, \quad i=1,2 .
\end{aligned}
$$

Proposition 2 Consider model (4.7) and assume that the initial distribution of the trait $\varphi(\mathbf{a})$ is

(i) normal with mean $m_{0}$ and variance $\sigma_{0}^{2}$. Then the trait distribution will also be normal at any $t$ with mean $m_{t}=m_{0}+\sigma_{0}^{2} q_{1}(t)$ and with the same variance $\sigma_{0}^{2}$;

(ii) Poisson with mean $m_{0}$. Then the trait distribution will also be Poisson at any $t$ with the mean $m_{t}=m_{0} \exp \left(q_{1}(t)\right)$;

(iii) $\Gamma$-distribution with the coefficients $k, a, \eta$, i.e. $P_{0}(\varphi=x)=a^{k}(x-\eta)^{k-1}$ $\exp (-(x-\eta) a) / \Gamma(k)$, where $k, a>0,-\infty<\eta<\infty, x \geq \eta ; \Gamma(k)$ is the $\Gamma$-function.

Define $T^{*}=\inf \left(t: q_{1}(t)=a\right)$, if such t exists, otherwise $T^{*}=T$. Then the trait $\varphi$ will be $\Gamma$ - distributed at any time moment $t<T^{*}$ with coefficients $a-q_{1}(t), k, \eta k$, such that $E^{t}[\varphi]=\eta+k /\left(a-q_{1}(t)\right), \sigma_{t}^{2}=k /\left(a-q_{1}(t)\right)^{2}$.

The list of practically implemented distributions can be extended.

Now let us consider model (4.7) with many traits when

$$
F(t, \mathbf{a})=\sum_{i=1}^{n} u_{i}\left(t, G_{i}\right) \varphi_{i}(\mathbf{a})
$$


The regulating functional for system (4.7), (4.10)

$$
\Phi(r ; \lambda)=\int_{A} r(\mathbf{a}) \exp \left(\sum_{i=1}^{n} \lambda_{i} \varphi_{i}(\mathbf{a})\right) P(0, \mathbf{a}) d \mathbf{a}
$$

and the auxiliary variables $q_{i}(t)$ solve the escort system:

$$
\begin{aligned}
d q_{i} / d t & =u_{i}\left(t, N(0) \Phi\left(g_{i} ; \mathbf{q}\right)\right), \\
q_{i}(0) & =0, \quad i=1, \ldots, n .
\end{aligned}
$$

If initially the traits $\varphi_{i}$ (a) are independent (as random variables on probabilistic space $\{A, \mathbf{A}, P(0, \mathbf{a})\})$ then they remain independent indefinitely (as random variables on probabilistic space $\{A, \mathbf{A}, P(t, \mathbf{a})\}$ for any $t)$ and given the initial mgf their joint mgf can be easily computed at any time moment (see Karev 2005b). In reality, the evolution of a system is governed by simultaneous selection over many traits, whose contributions to fitness depend on each other. The evolution of the pdf of the vector $\boldsymbol{\varphi}=\left(\varphi_{1}, \ldots, \varphi_{n}\right)$ in the general case of correlated traits $\left\{\varphi_{i}, i=1, \ldots, n\right\}$ is of great practical interest. Let us recall some definitions (see Kotz et al. 2000).

A random vector $\mathbf{X}=\left(X_{1}, \ldots, X_{n}\right)$ has a multivariate normal distribution with the mean $E \mathbf{X}=\mathbf{m}=\left(m_{1}, \ldots, m_{n}\right)$ and covariance matrix $\mathbf{C}=\left\{c_{i j}\right\}, c_{i j}=\operatorname{cov}\left(X_{i}, X_{j}\right)$ if its mgf is $M(\lambda)=E\left[\exp \left(\lambda^{T} \mathbf{X}\right)\right]=\exp \left(\lambda^{T} \mathbf{m}+1 / 2 \lambda^{T} \mathbf{C} \lambda\right)$.

A random vector $\mathbf{X}=\left(X_{1}, \ldots, X_{n}\right)$ has a multivariate polynomial distribution with parameters $\left(k ; p_{1}, \ldots, p_{n}\right)$, if $P\left(X_{1}=m_{1}, \ldots, X_{n}=m_{n}\right)=\frac{k !}{m_{1} ! \ldots, m_{n} !} p_{1}^{m_{1}} \ldots p_{n}^{m_{n}}$ for $\sum_{i=1}^{n} m_{i}=k$. The mgf of the polynomial distribution is $M(\lambda)=\left(\sum_{i=1}^{n} p_{i} \exp \left(\lambda_{i}\right)\right)^{k}$.

A general class of multivariate natural exponential distributions is especially important for selection systems and their applications. It includes multivariate polynomial, normal, and other distributions as special cases. A random $n$-dimension vector $\mathbf{X}=\left(X_{1}, \ldots, X_{n}\right)$ has multivariate natural exponential distribution (NED) with parameters $\boldsymbol{\theta}=\left(\theta_{1}, \ldots, \theta_{n}\right)$ with respect to the positive measure $v$ on $R^{n}$ if its joint density function is of the form $f(\mathbf{X})=h(\mathbf{X}) \exp \left(\mathbf{X}^{T} \boldsymbol{\theta}-s(\boldsymbol{\theta})\right)$ where $s(\boldsymbol{\theta})$ is the normalization function. The mgf of NED is

$$
M(\boldsymbol{\lambda})=E_{\nu}\left[\exp \left(\lambda^{T} \mathbf{X}\right)\right]=\exp (s(\boldsymbol{\theta}+\lambda)-s(\boldsymbol{\theta}))
$$

Proposition 3 Let us assume that at the initial time moment the vector $\varphi=\left(\varphi_{1}, \ldots \varphi_{n}\right)$ has

i) multivariate normal distribution with the mean vector $\mathbf{m}(0)$ and covariance matrix $\mathbf{C}=\left(c_{i j}\right)$. Then the vector $\boldsymbol{\varphi}$ also has the multivariate normal distribution at any moment $t<T$ with the same covariance matrix $\mathbf{C}$ and the mean vector $\mathbf{m}(t), m_{i}(t)=m_{i}(0)+1 / 2 \sum_{k=1}^{n}\left(c_{i k}+c_{k i}\right) q_{k}(t)$;

ii) multivariate polynomial distribution. Then the vector $\varphi$ also has the multivariate polynomial distribution at any moment $t<T$ with parameters $\left(k ; p_{1}(t), \ldots\right.$, $\left.p_{n}(t)\right)$ where $p_{i}(t)=p_{i} \exp \left(q_{i}(t)\right) / \sum_{j=1}^{n} p_{j} \exp \left(q_{j}(t)\right)$; 
iii) multivariate natural exponential distribution on $R^{n}$ with respect to the Lesbegue measure, with the density function $f_{0}(\mathbf{X})=h(\mathbf{X}) \exp \left(\mathbf{X}^{T} \boldsymbol{\theta}-s(\boldsymbol{\theta})\right)$ and the $m g f$ $M(\boldsymbol{\lambda})=\exp (s(\boldsymbol{\theta}+\boldsymbol{\lambda})-s(\boldsymbol{\theta}))$. Then the vector $\boldsymbol{\varphi}$ also has the multivariate NED at any moment $t<T$ with the parameters $\boldsymbol{\theta}+\mathbf{q}(t)$, the density function $f_{t}(\mathbf{X})=h(\mathbf{X}) \exp \left(\mathbf{X}^{T}(\boldsymbol{\theta}+\mathbf{q}(t))-s(\boldsymbol{\theta}+\mathbf{q}(t))\right)$ and the moment generating function $M_{t}(\boldsymbol{\lambda})=\exp (s(\boldsymbol{\theta}+\boldsymbol{\lambda}+\mathbf{q}(t))-s(\boldsymbol{\theta}+\mathbf{q}(t)))$.

Proofs of Propositions 2, 3 are similar to that given in Karev (2005b) for a less general model. Both assertions up to the definition of the auxiliary variables are valid for selection systems whose fitness may depend on some intensive regulators.

\section{Applications}

The simplest selection system is the inhomogeneous Malthusian-like model $d l(t, \mathbf{a}) /$ $d t=l(t, \mathbf{a}) \varphi(\mathbf{a})$. The function $\varphi(\mathbf{a})$ itself can be often considered as a distributed parameter, and then the model reads $d l(t, x) / d t=x l(t, x)$.

Let $M(\lambda)=\int_{A} \exp (\lambda x) P_{0}(x) d x$ be the mgf of the initial distribution of the parameter $x$. Then the solution of the model $l(t, x)=\exp (x t) l(0, x)$ and $N(t)=N_{0} M(t)$. The model distribution solves the replicator equation $d P_{t}(x) / d t=P_{t}(x)\left(x-E^{t}[x]\right)$; its solution is $P_{t}(x)=P_{0}(x) \exp (x t) / M(t)$.

Inhomogeneous Malthusian models and their applications to some problems of forest ecology and global demography were studied in Karev (2000, 2003, 2005a). It was shown that even this simplest inhomogeneous model possess a variety of solutions depending on the initial distribution, which may have many interesting and even counterintuitive peculiarities. Let us demonstrate some of them on the example of inhomogeneous Malthusian-like model with limiting factors.

Example 1 Principle of limiting factors, according to Liebig (1876), states that at any given moment the rate of a process is determined by the factor whose sufficiently small modification produces a change of the rate; it is assumed that similar changes in other factors do not affect the rate (see Poletaev 1966 for mathematical formulation). The principle of limiting factors was actually used in a model of early biological evolution suggested in Zeldovich et al. (2007). Each organism was characterized by the vector a where the component $a_{i}$ is the thermodynamic probability that protein $i$ is in its native conformation. In order to study the connection between molecular evolution and population, the authors suppose that the organism death rate $d$ depends on the stability of its proteins as $d=d_{0}\left(1-\min a_{i}\right), d_{0}=b /\left(1-a_{0}\right), b$ is the birth rate, $a_{0}$ is the native state probability of a protein. Hence, neglecting possible mutations (accounted for by the authors in their simulations), the model can be formalized as the system

$$
\left.d l(t, \mathbf{a}) / d t=l(t, \mathbf{a}) d_{0}\left(m(\mathbf{a})-a_{0}\right)\right)
$$

where $m(\mathbf{a})=\min \left[a_{1}, \ldots, a_{n}\right]$. In what follows we let $d_{0}=1$ for simplicity. It was supposed in Zeldovich et al. (2007) that the values $a_{i}$ are independent from each other and have the Boltzmann distribution. We can consider $a_{i}$ as the $i$-th realization of 
a random variable with a common pdf $f(a)$. Let $G(a)=\int_{0}^{a} f(x) d x$ be the cumulative distribution function. Then, it is well known that the pdf of $\min \left[a_{1}, \ldots a_{n}\right]$ is equal to $g(a)=n(1-G(a))^{n-1} f(a)$. Equation (5.1) is a version of the inhomogeneous Malthusian equation, which can now be solved explicitly at any given pdf $f(a)$. In particular, if

$$
f(a)=\exp (-a / T) / Z, \quad Z=\sum_{a} \exp (-a / T)
$$

is the Boltzmann distribution with $a>0$, then

$$
\begin{aligned}
g(a) & =n(1-G(a))^{n-1} f(a) \\
& \left.=n\left(\sum_{x>a} \exp (-x / T)\right)^{n-1} \exp (-a / T) / \sum_{x} \exp (-x / T)\right) .
\end{aligned}
$$

For distribution (5.2) with continuous range of values of $a, a \in(0, \infty), Z=T$, $1-G(x)=\exp (-x / T)$ and

$$
g(a)=n(\exp (-a(n-1) / T) \exp (-a / T) / T=n / T \exp (-a n / T)
$$

If $a \in(0, E)$, then $Z=T(1-\exp (-E / T)), 1-G(a)=\frac{\exp ((E-a) / T)-1}{\exp (E / T)-1}$, and

$$
g(a)=\frac{n \exp (-a / T)}{T(1-\exp (-E / T))}\left[\frac{1-\exp ((E-a) / T)}{1-\exp (E / T)}\right]^{n-1} .
$$

Let $M_{0}(\lambda)=E^{0}[\exp (\lambda m)]$. For initial distribution $(5.3), M_{0}(\lambda)=\frac{1}{1-\lambda T / n}$. Hence,

$$
\begin{aligned}
l(t, \mathbf{a}) & =l(0, \mathbf{a}) \exp \left(\left(m(\mathbf{a})-a_{0}\right) t\right), \\
N(t) & =N(0) \exp \left(-a_{0} t\right) \frac{1}{1-t T / n}, \\
P(t, \mathbf{a}) & =P(0, \mathbf{a}) \exp (m(\mathbf{a}) t)(1-t T / n) .
\end{aligned}
$$

At the moment $t_{\max }=n / T$ the population "blows up": $N(t)$ tends to infinity as $t \rightarrow t_{\max }$. Let us denote $p(t, a)=P(t,\{\mathbf{a}: m(\mathbf{a})=a\})$. Then at $t<t_{\max }$

$$
p(t, a)=n / T \exp (-a n / T+a t)(1-t T / n)=(n / T-t) \exp (a(t-n / T)) .
$$

The probability $P(t,\{\mathbf{a}: m(\mathbf{a})<a\})$ tends to 0 for any finite $a$ as $t \rightarrow t_{\max }$. Loosely speaking, the total "probability mass" goes to infinity after a finite time interval. So, we should conclude that model (5.1), (5.2) which allow arbitrary large values of the parameter $a$ with nonzero probability have no "physical" sense.

This problem can be eliminated by taking the initial distribution (5.4), which allows only bounded values of the parameter $a$. For pdf (5.4), the integral $M_{0}(\lambda)=$ 
$\int_{0}^{E} \exp (\lambda x) g(x) d x$ is finite for any $\lambda$; although it cannot be expressed in quadratures we can obtain much information about the system distribution and its dynamics. The current pdf

$$
p(t, a)=\frac{n}{T(\exp (E / T)-1)^{n}} \exp ((E-a) / T)(\exp ((E-a) / T)-1)^{n-1} \frac{\exp (a t)}{M_{0}(t)}
$$

where $M_{0}(t)$ is finite for all $t$. So, the pdf is well defined at any time moment, in contrast to the previous case. The total distribution concentrates with time at the point $a=E$, which provides the maximal reproduction rate. Let us emphasize that the pdf $p(t, a)$ does not depend on the native state probability $a_{0}$.

Example 1 demonstrates a possibility of the "blowing up" phenomenon, when the total population size goes to infinity after a finite time interval. Similar phenomenon was discovered earlier in models of global demography Karev (2005a). One may suppose that the choice of the Malthusian model for population dynamics is to blame for this phenomenon and the problem should disappear if the Malthusian model is replaced by the logistic model. Surprisingly, it is not the case for inhomogeneous logistic models. Let us apply our approach to a wide class of generalized inhomogeneous logistictype models:

$$
\begin{aligned}
d l(t, \mathbf{a}) / d t & =l(t, \mathbf{a}) F(t, \mathbf{a}), \\
F(t, \mathbf{a}) & =f_{1}(t) b(\mathbf{a})-f_{2}(t) d(\mathbf{a})
\end{aligned}
$$

where $f_{1}(t) b(\mathbf{a})$ is the birth rate, $f_{2}(t) d(\mathbf{a})$ is the death rate, and $f_{i}$ are functions of the regulators $H(t)=E^{t}[h]$ or $G(t)=N(t) E^{t}[g]$. Different versions of equation (5.5) were discussed in numerous works. Theorems of existence and uniqueness and asymptotic behavior of some equations of the form (5.5) were studied in Ackleh et al. (1999, 2005), Gorban (1984, 2007), Perthame (2007).

The method described in Sect. 4.2 allows us to obtain the solution of equation (5.5) at any instant within the time interval where the global solution of the escort system exists. We will not rewrite the general formulas in terms of equation (5.5); instead, we give the solutions of some particular inhomogeneous logistic equations used (but not solved explicitly) in the literature.

Example 2 The following example of the system with inheritance was considered in Gorban (2007):

$$
\begin{aligned}
d l(t, \mathbf{a}) / d t & =l(t, \mathbf{a}) F(t, \mathbf{a}), \\
F(t, \mathbf{a}) & =b(\mathbf{a})-\int_{A} m(\mathbf{a}) l(t, \mathbf{a}) d \mathbf{a}=b(\mathbf{a})-N(t) E^{t}[m] .
\end{aligned}
$$

It can be interpreted as follows. Let $b(\mathbf{a})$ be the specific birth rate of inherited varieties a. The death rate is determined by the common factor $\int_{A} m(\mathbf{a}) l(t, \mathbf{a}) d \mathbf{a}$ where $m(\mathbf{a})$ is the individual contribution of variety a to this death rate. Using the method summarized above, we are now able to give an explicit solution of this model. Compose 
the generating functional $\Phi\left(r ; \lambda_{1}, \lambda_{2}\right)=\exp \left(-\lambda_{2}\right) E^{0}\left[r \exp \left(\lambda_{1} b\right)\right]$. Then the escort system reads

$$
\begin{aligned}
& d q_{1} / d t=1, q_{1}(0)=0, \quad \text { hence } q_{1}(t)=t \\
& d q_{2} / d t=N(0) \Phi\left(m ; q_{1}, q_{2}\right)=N(0) \exp \left(-q_{2}\right) E^{0}[m \exp (t b)], \quad q_{2}(0)=0 .
\end{aligned}
$$

This equation can be easily integrated since $E^{0}[m \exp (t b)]=f(t)$ is a known function of $t$ at given $P(0, \mathbf{a}): \exp \left(q_{2}(t)\right)=1+N(0) \int_{0}^{t} f(s) d s=1+N(0) E^{0}\left[\frac{m}{b}(\exp (t b)-1)\right]$.

The solution to the model

$$
\begin{aligned}
l(t, \mathbf{a}) & =l(0, \mathbf{a}) \exp \left(t b(\mathbf{a})-q_{2}(t)\right) \\
& =l(0, \mathbf{a}) \exp (t b(\mathbf{a})) /\left\{1+N(0) E^{0}\left[\frac{m}{b}(\exp (t b)-1)\right]\right\} .
\end{aligned}
$$

Also, $N(t)=N(0) E^{0}[\exp (t b)] /\left\{1+N(0) E^{0}\left[\frac{m}{b}(\exp (t b)-1)\right]\right\}$, and

$$
P(t, \mathbf{a})=l(t, \mathbf{a}) / N(t)=P(0, \mathbf{a}) \exp (t b(\mathbf{a})) / E^{0}[\exp (t b)] .
$$

Remark, that the current pdf $P(t, \mathbf{a})$ does not depend on the death rate. We can observe now an interesting phenomenon, which is impossible for "homogeneous" logistic models. Let us suppose that the birth rate $b(\mathbf{a})$ is $\Gamma$-distributed at the initial moment, i.e. $P_{0}(b=x)=T^{k} x^{k-1} \exp (-x T) / \Gamma(k)$, where $k, T>0$ are constants, $x \geq 0$.

Then $E^{0}[\exp (t b)]=(1-t / T)^{-k}$ for $t<T$. So, $P(t,\{b<x\})<\exp (t x)(1-$ $t / T)^{k} \rightarrow 0$ as $t \rightarrow T$ for any $x$; by words, the frequency of individuals with a finite birth rate vanishes as $t \rightarrow T$.

Example 3 There exist a large number of papers devoted to the problem of evolution of altruism. We do not discuss here an interesting and important problem how an altruistic trait can be selected. Instead, using the developed tools, we give an exact solution of a model used in Wilson and Dugatkin (1997). Consider a population in which each individual possesses a trait $x$ that increases the fitness of everyone in the population (including itself) by an amount $m x$ at a personal cost $-c x$. Then the fitness of the individual is

$$
F(t, x)=-c x+m N(t) E^{t}[x], \quad x>0,
$$

which coincides with the fitness of Example 2 up to notation but has the opposite sign. It is interesting that model (5.7) exhibits essentially different dynamical behavior. Using formula (5.6) we can write the model solution as

$$
\begin{aligned}
l(t, x) & =l(0, x) \exp (-t c x) /\left\{1+N(0) m / c\left(E^{0}[\exp (-t c x)]-1\right\},\right. \\
N(t) & =N(0) E^{0}[\exp (-t c x)] /\left\{1+N(0) m / c E^{0}[\exp (-t c x)]-1\right\}, \quad \text { and } \\
P(t, x) & =P(0, x) \exp (-t c x) / E^{0}[\exp (-t c x)] .
\end{aligned}
$$

As $c>0$, the model solution exists and is finite indefinitely for any initial distribution of the trait. 
Example 4 A microbial population in an environment of an antimicrobial agent was studies in Nikolaou and Tam (2005); the suggested model has (up to notation) the form of inhomogeneous Malthusian equation

$$
d N / d t=(K-m(C)) N
$$

where $K$ is the physiological growth rate, $m(C)$ is the kill rate induced by the antimicrobial agent, which has concentration $C$. A more accurate version with logistic growth rate was also discussed:

$$
d N / d t=K N(1-N / B)-m(C) N .
$$

The value of $m(C)$, in comparison to $K$, represents the resistance of microbes to a specific antimicrobial agent with concentration $C$. Population resistance is distributed over a multitude of values. For the Malthusian version of the model, (5.9), the authors derive the equation for the size of an entire population over time and then approximate it using the variance and higher-order cumulants of distribution of the kill rate over a heterogeneous population.

The theory of inhomogeneous Malthusian and logistic equations developed earlier Fisher (1999), Karev (2005a) allows one to obtain complete solutions of equations (5.9), (5.10). Letting $R=K-m(C)$ to be the resistance of microbes to the antimicrobial agent at concentration $C$, we can consider $R$ as the parameter distributed over the population. The distribution of $R$ can be easily computed if the distribution of concentration $C$ is known. The inhomogeneous model is of the form $d l(t, R) / d t=R l(t, R)$ where $l(t, R)$ is the population density over the resistance $R$. Let $M_{0}(\lambda)=\int_{A} \exp (\lambda R) P(0, R) d R$ be the mgf of the initial distribution of resistance. Then the population size at moment $t \quad N(t)=N(0) M_{0}(t)$, and the current pdf of resistance $P(t, R)=P(0, R) \exp (t R) / M_{0}(t)$.

For example, if we suppose (as in Nikolaou and Tam 2005) that the initial distribution of the resistance is normal with a mean $m_{0}$, variance $\sigma_{0}^{2}$, and mgf $M_{0}(\lambda)=$ $\exp \left(\lambda^{2} \sigma_{0}^{2} / 2+\lambda m_{0}\right)$, then the resistance distribution is also normal at any $t$ with the mean $E^{t}[R]=m_{0}+\sigma_{0}^{2} t$ and with the same variance $\sigma_{0}^{2}$, and $N(t)=$ $N(0) \exp \left(t^{2} \sigma_{0}^{2} / 2+t m_{0}\right)$. Remark, that this supposition is not realistic as the resistance should be positive. If the initial distribution of the resistance is $\Gamma$-distribution, $P(0, R)=s^{k}(R-\eta)^{k-1} \exp (-(R-\eta) s) / \Gamma(k)$, with mean $E^{0}[R]=\eta+k / s$, variance $\sigma_{0}^{2}=k / s^{2}$, and the $\operatorname{mgf} M_{0}(\lambda)=\exp (\lambda \eta) /(1-\lambda / s)^{k}$ for $\lambda<s$, then $R$ is also $\Gamma$-distributed at any moment $t<s$ with mean $E^{t}[R]=\eta+k /(s-t)$ and variance $\sigma_{t}^{2}=k /(s-t)^{2}$, and $N(t)=N(0) \exp (t \eta) /(1-t / s)^{k}$. The model "blows up" when $t=s$, i.e. the population size tends to infinity as $t \rightarrow s$ and hence the model is unrealistic.

The normal and $\Gamma$-distributions are completely characterized by their mean and variance; it follows from the examples given above that the fate of the population can be dramatically different at the same initial mean and variance of the resistance.

More realistically, the actual initial distribution of the resistance should be concentrated in a finite interval; we can suppose that the initial distribution is uniform, Beta-distribution, or truncated exponential in that interval. In all these cases the model 
can be solved explicitly. For example, in the last case $P(0, R)=V \exp (-s R)$ where $0 \leq R \leq c=$ const, $s$ is the distribution parameter, and $V=s /(1-\exp (-s c))$ is the normalization constant. Then the current population size $N(t)$ is defined by the formula $N(t)=N(0)(1-t / s)^{-1}(1-\exp (c(t-s))) /(1-\exp (-s c))$ and the current distribution of $R$ is the truncated exponential distribution with the parameter $s-t$ (see Karev 2005a for details).

Next, let us consider a more realistic logistic version (5.10) of the model. Corresponding inhomogeneous model reads

$$
d l(t, C) / d t=l(t, C)(K(1-N(t) / B)-m(C))
$$

where $l(t, C)$ is the microbial subpopulation under the pressure of antimicrobial agent with concentration $C$. The reproduction rate depends only on the total population size, so in order to solve this equation we only need to know the mgf of the initial distribution $P(0, C)$ (see Sects. 4.2, 4.3)). Let

$M_{0}(\lambda, \delta)=\int_{A} \exp (\lambda+\delta m(C)) P(0, C) d C=\exp (\lambda) E^{0}[\exp (\delta m)]$. Then the escort system for auxiliary variables read (see (4.5)):

$$
\begin{aligned}
& d s / d t=-1, s(0)=0, \text { hence } s(t)=-t \\
& d q / d t=K\left(1-N(0) \exp (q) E^{0}[\exp (-t m)] / B\right)=K(1-A(t) \exp (q)), q(0)=0 .
\end{aligned}
$$

Here we denote $A(t)=N(0) E^{0}[\exp (-t m)] / B$. The function $A(t)$ is known at a given initial distribution and hence equation (5.12) can be solved (at least, numerically).

The solution to model (5.11) is $l(t, C)=l(0, C) \exp (q(t)-\operatorname{tm}(C))$; the total population size $N(t)=N(0) \exp (q(t)) E^{0}[\exp (-t m)]$; the distribution of the agent concentration at moment $t$ is $P(t, C)=P(0, C) \exp (-t m(C)) / E^{0}[\exp (-t m)]$.

The model and its solution are simplified if we consider the kill rate $m$ rather then the concentration $C$ as a distributed parameter. In this case the model reads

$$
d l(t, m) / d t=l(t, m)(K(1-N(t) / B)-m) .
$$

Let $M_{0}(\lambda)=E^{0}[\exp (\lambda m)]$ be the mgf of initial distribution of the kill rate $m$. Changing the variable, $z=\exp (q)$, we obtain the equation $d z / d t=z K(1-$ $\left.z N(0) M_{0}(-t) / B\right), z(0)=1$. This equation can be solved at the given mgf $M_{0}$ of the initial distribution.

The solution to model (5.13) is now $l(t, m)=l(0, m) z(t) \exp (-t m)$; the total population size $N(t)=N(0) z(t) M_{0}(-t)$, and the distribution of the kill rate $P(t, m)=$ $P(0, m) \exp (-t m) / M_{0}(-t)$.

Example 5 Many particular models have the form of the inhomogeneous logistic equation

$$
d l(t ; \beta, \mu) / d t=l(t ; \beta, \mu)\left[\left(\beta f_{1}(N(t))-\mu f_{2}(N(t))\right]\right.
$$


(see, e.g., Ackleh et al. 2005 and references therein). Let us give explicit formulas for the solution of this equation. Let $M_{0}\left(\lambda_{1}, \lambda_{2}\right)=E^{0}\left[\exp \left(\lambda_{1} \beta+\lambda_{2} \mu\right)\right]$ be the mgf of the joint initial distribution of $\beta$ and $\mu$. The escort system for auxiliary variables reads

$$
\begin{aligned}
& d q_{1} / d t=f_{1}\left(N(0) M_{0}\left(q_{1}, q_{2}\right)\right), q_{1}(0)=0 ; \\
& d q_{2} / d t=-f_{2}\left(N(0) M_{0}\left(q_{1}, q_{2}\right)\right), q_{2}(0)=0 .
\end{aligned}
$$

The solution to equation (5.14) is

$$
l(t ; \beta, \mu)=l(0 ; \beta, \mu) \exp \left(q_{1}(t) \beta+q_{2}(t) \mu\right),
$$

the total population size is given by

$$
N(t)=N(0) M_{0}\left(q_{1}(t), q_{2}(t)\right)
$$

and the current distribution is given by the formula

$$
P(t ; \beta, \mu)=P(0 ; \beta, \mu) \exp \left(q_{1}(t) \beta+q_{2}(t) \mu\right) / M_{0}\left(q_{1}(t), q_{2}(t)\right) .
$$

A particular case of equation (5.14)

$$
d l(t ; \beta, \mu) / d t=l(t ; \beta, \mu)(\beta-\mu N(t))
$$

was studied in Ackleh et al. (1999) for independent growth and mortality parameters, $\beta$ and $\mu$, uniformly distributed in the intervals $\left[a_{1}, b_{1}\right]$ and $\left[a_{2}, b_{2}\right]$ accordingly. The theorem of existence and uniqueness was established; it was also proven that the population concentrates asymptotically in the parametric point $\left[b_{1}, a_{2}\right]$ with the highest growth to mortality ratio. Remark that both conditions, the independence of the parameters and boundedness of their domains of values are essential for the last statement to be valid. For example, if $\beta=c \mu$ then the population does not concentrate in a parametric point but stays inhomogeneous indefinitely Karev (2005a). The second condition is discussed below.

Let us solve equation (5.18). The first equation of the escort system (5.15) reads $d q_{1} / d t=1, q_{1}(0)=0$, hence $q_{1}(t)=t$. So, the second equation is $d q_{2} / d t=$ $-N(0) M_{0}\left(t, q_{2}\right), q_{2}(0)=0$.

Let the parameters $\beta \in\left[a_{1}, b_{1}\right]$ and $\mu \in\left[a_{2}, b_{2}\right]$ be independent and uniformly distributed at the initial moment, i.e. $P(0 ; \beta, \mu)=1 /\left(\left(b_{1}-a_{1}\right)\left(b_{2}-a_{2}\right)\right)$. The mgf of the uniform distribution in $[a, b]$ is $M_{0}(\lambda)=(\exp (\lambda b)-\exp (\lambda a)) /(\lambda(b-a))$, hence

$$
\begin{aligned}
M_{0}\left(\lambda_{1}, \lambda_{2}\right)= & E^{0}\left[\exp \left(\lambda_{1} \beta+\lambda_{2} \mu\right)\right] \\
= & \left(\exp \left(\lambda_{1} b_{1}\right)-\exp \left(\lambda_{1} a_{1}\right)\right) /\left(\lambda_{1}\left(b_{1}-a_{1}\right)\right)\left(\exp \left(\lambda_{2} b_{2}\right)\right. \\
& \left.-\exp \left(\lambda_{2} a_{2}\right)\right) /\left(\lambda_{2}\left(b_{2}-a_{2}\right)\right)
\end{aligned}
$$


Then the auxiliary variable solves the equation

$$
\begin{aligned}
d q_{2} / d t & =-N(0) M_{0}\left(t, q_{2}\right) \\
& =-N(0) \frac{\exp \left(t b_{1}\right)-\exp \left(t a_{1}\right)}{t\left(b_{1}-a_{1}\right)} \frac{\exp \left(q_{2}(t) b_{2}\right)-\exp \left(q_{2}(t) a_{2}\right)}{q_{2}(t)\left(b_{2}-a_{2}\right)}
\end{aligned}
$$

The solution of equation (5.18) is then

$$
l(t ; \beta, \mu)=1 /\left(\left(b_{1}-a_{1}\right)\left(b_{2}-a_{2}\right)\right) \exp \left(t \beta+q_{2}(t) \mu\right) .
$$

Next, $P(t ; \beta, \mu)=P^{1}(t ; \beta) P^{2}(t ; \mu)$ where

$$
P^{1}(t ; \beta)=\frac{\exp (t \beta)}{\left(b_{1}-a_{1}\right) E^{0}[\exp (t \beta)]}, \quad P^{2}(t ; \mu)=\frac{\exp \left(q_{2}(t) \mu\right)}{\left(b_{2}-a_{2}\right) E^{0}\left[\exp \left(q_{2}(t) \mu\right)\right]} .
$$

It is now easy to see (taking into account that $q_{2}(t) \rightarrow-\infty$ as $t \rightarrow \infty$ ) that $P_{1}(t ; \beta)$ and $P_{2}(t ; \mu)$ in course of time concentrate at points $b_{1}$ and $a_{2}$ correspondingly, in accordance with Ackleh et al. (1999).

The following example shows that qualitatively different asymptotical behavior of the same equation is possible with another initial distribution. Let the positive parameters $\beta, \mu$ be independent again, and the initial distribution of both parameters be exponential, $P_{i}(x)=s_{i} \exp \left(-x s_{i}\right)$, with the $\operatorname{mgf} M_{i}(\lambda)=1 /\left(1-\lambda / s_{i}\right), i=1,2$. Let us put $s_{1}=T, s_{2}=1$, and $N(0)=1$ for simplicity. Then $M_{0}\left(\lambda_{1}, \lambda_{2}\right)=\frac{1}{\left(1-\lambda_{1} / T\right)\left(1-\lambda_{2}\right)}$, and the auxiliary variable solves the equation

$$
d q_{2} / d t=-N(0) M_{0}\left(t, q_{2}\right)=-\frac{1}{(1-t / T)\left(1-q_{2}\right)}, \quad q_{2}(0)=0,
$$

which can be easily integrated: $q_{2}(t)=1-\sqrt{1-2 T \ln (1-t / T)}$.

Now we can see that the global solution of equation (5.19) exists only at $t<T$ and $q_{2}(t) \rightarrow-\infty$ as $t \rightarrow T$. It means that the solution of the inhomogeneous logistic equation (5.18) with exponentially distributed parameters exists only up to the moment $t=T$. The model solution $l(t ; \beta, \mu)$, the total population size and the parameter distributions for $t<T$ can be written down with the help of formulas (5.16)-(5.17). In particular, $N(t)=N(0) /\{(1-t / T) \sqrt{1-2 T \ln (1-t / T)}\}$. As $t \rightarrow T$, the total population size tends to infinity and the population vanishes in any finite interval of values for both parameters, $\beta$ and $\mu$. Remark, that a similar phenomenon of "population explosion" at a certain time moment $T<\infty$ is realized for a wide class of $\Gamma$-distributed parameters.

Example 6 To conclude this section, let us demonstrate how to solve the inhomogeneous version of the well-known Ricker equation (see, e.g., Thieme 2003, Sect. 5.3)):

$$
d l(t ; \beta, \mu) / d t=l(t ; \beta, \mu)[(\beta \exp (-c N(t))-\mu)] .
$$


Let $M_{0}\left(\lambda_{1}, \lambda_{2}\right)$ be the mgf of the joint initial distribution of $\beta$ and $\mu$. Then the escort system reads

$$
\begin{aligned}
& d q_{2} / d t=-1, q_{2}(0)=0, \quad \text { hence } q_{2}(t)=-t \\
& d q_{1} / d t=\exp \left(-c N(0) M_{0}\left(q_{1},-t\right)\right), \quad q_{1}(0)=0 .
\end{aligned}
$$

Equation (5.21) can be solved at known $M_{0}$ and then the solution to (5.20) is equal to $l(t ; \beta, \mu)=l(0 ; \beta, \mu) \exp \left(q_{1}(t) \beta-t \mu\right)$; the total population size $N(t)=N(0) M_{0}$ $\left(q_{1}(t),-t\right)$ and the system distribution $P(t ; \beta, \mu)=P(0 ; \beta, \mu) \exp \left(q_{1}(t) \beta-t \mu\right) /$ $M_{0}\left(q_{1}(t),-t\right)$.

For example, let the parameters $\beta$ and $\mu$ be independent and exponentially distributed in $[0, \infty)$ with the means $s_{1}$ and $s_{2}$ at the initial instant. Then $M_{0}(q,-t)=$ $s_{1} s_{2} /\left(\left(s_{1}-q\right)\left(s_{2}+t\right)\right)$, and equation (5.21) for the auxiliary variable reads $d q_{1} / d t=$ $\exp \left(-c N(0) s_{1} s_{2} /\left(\left(s_{1}-q_{1}\right)\left(s_{2}+t\right)\right), q_{1}(0)=0\right.$.

This equation has a stable state $q_{1}=s_{1}$. As $t \rightarrow \infty, q_{1}(t) \rightarrow s_{1}$, the total population size tends to infinity and the population density concentrates at the value $\mu=0$ of the parameter $\mu$ and vanishes in any finite interval of values of the parameter $\beta$.

\section{Discussion}

Mathematical theory of selection has a long history; R. Fisher, S. Wright, J. Haldane were its father-founders. G. Price was the first who tried to find a general formulation of selection that could be applied to any (not only biological) problem and to develop a formal general theory. He hoped that the concept of selection proposed in his paper Price (1995), which was published only in 1995, "will contribute to the future development of 'selection theory' as helpfully as Hartley's concept of information contributed to Shannon's communication theory. ... Many scientists must have felt surprise to find that at so late date there had still remained an opportunity to develop so fundamental a scientific area. Perhaps a similar opportunity exists today in respect to selection theory".

The Price equation was an outstanding contribution to the future theory; its particular cases are the Fisher fundamental theorem and the covariance equation. The Price equation is universally applicable to any selection systems at any instant independently from the underlying mathematical model and its specific dynamics (see, e.g., Rice 2006, ch.6 for details) because this equation is a mathematical identity. It is a reason for the theoretical universality and practical unavailing ("dynamical insufficiency") of the Price equation.

The Haldane optimal principle can be considered as one of the first general assertions about selection systems; it describes the asymptotical behavior of a population composition. This principle was generalized in Semevsky and Semenov (1982), ch.3 for models with discrete time and in Gorban (1984) (Appendix) for models with continuous time. The authors developed an abstract theory of systems with inheritance 
and applied it to some problems of mathematical biology (see, e.g., monograph Gorban 1988 and the survey Gorban 2007). The "resampling down" of the initial variety demonstrates the qualitative effects of "natural selection" as $t \rightarrow \infty$. The theory gives a complete description of the support of the limit distributions but the dynamics of systems on a finite time interval is out of the scope of this theory.

Thus, at the present time the mathematical theory of selection has general frameworks for mathematical modeling (the systems with inheritance, replication equations, selection systems), some fundamental assertions (like the Haldane principle, the Fisher fundamental theorem of selection and the Price equations) and many particular models of inhomogeneous populations and communities. Most of these models have a form of many- or infinite dimensional differential or difference systems of equations. Some theorems of existence and uniqueness and asymptotic behavior of solutions to particular classes of such equations were established (see, e.g., Ackleh et al. 1999; Gorban 2007; Perthame 2007), but no general methods for solving the models analytically are known, except for numerical investigation.

In this paper we suggest a general approach to a wide class of self-regulated selection systems. The main evolutionary forces in an evolving system are selection, mutation and random drift; it should be noticed that our approach explicitly examines only selection. The developed theory allows one to reduce the complex inhomogeneous models to the "escort systems" of ODEs that, in many cases, can be investigated analytically. Notice that even if the analytical solution of the escort system is not available, numerical solving the Cauchy problem for a system of ODE is much simpler than studying the initial problem numerically. It allows us to compute (in many cases, explicitly) the evolution of distributions and all statistical characteristics of interest of the initial selection system. Similar approach to discrete-time models was developed in Karev (2008). The considered examples show how different the global dynamics of a selection system can be depending on the initial distribution even for the same dynamical model.

We have systematically applied our approach to inhomogeneous Malthusian and logistic equations; explicit solutions to different models of these types used in the literature were derived. Analytical solutions of the considered models can provide new biological insights beyond the computer simulations performed in the original papers. We believe that derived explicit solutions may be helpful and necessary in order to be able to completely study corresponding models, which belong to different areas of mathematical biology; however, that is out of the scope of this paper. Applications to forest ecology modeling Karev (2003), global demography Karev (2005a), cancer modeling Karev et al. (2006), and epidemics in heterogeneous populations Novozhilov (2009) were published recently. It is our hope that the developed approach has potential for different applications these and others areas of science.

Acknowledgments This research was supported [in part] by the Intramural Research Program of the NIH, National Library of Medicine. The author thanks Dr. E. Koonin, Dr. F. Berezovsky, Dr. A. Novozhilov and anonymous reviewer for valuable comments.

Open Access This article is distributed under the terms of the Creative Commons Attribution Noncommercial License which permits any noncommercial use, distribution, and reproduction in any medium, provided the original author(s) and source are credited. 


\section{Appendix}

Proof of Theorem 1 Let $\{\mathbf{q}(t), \mathbf{s}(t)\}$ be a solution of Cauchy problem (3.5)-(3.6); denote for instant

$$
\begin{aligned}
K_{t}^{*}(\mathbf{a}) & =\exp \left(\sum_{i=1}^{n} q_{i}(t) \varphi_{i}(\mathbf{a})+\sum_{k=1}^{m} s_{k}(t) \psi_{k}(\mathbf{a})\right), \\
l^{*}(t, \mathbf{a}) / d t & =l(0, \mathbf{a}) K_{t}^{*}(\mathbf{a}), \\
N^{*}(t) & =N(0) \Phi(1 ; \mathbf{q}(t), \mathbf{s}(t)), \\
G_{i}^{*}(t) & =N(0) \Phi\left(g_{i} ; \mathbf{q}(t), \mathbf{s}(t)\right), \\
H_{i}^{*}(t) & =\Phi\left(h_{i} ; \mathbf{q}(t), \mathbf{s}(t)\right) / \Phi(1 ; \mathbf{q}(t), \mathbf{s}(t)), \\
F^{*}(t, \mathbf{a}) & =\sum_{i=1}^{n} u_{i}\left(t, G_{i}^{*}\right) \varphi_{i}(\mathbf{a})+\sum_{k=1}^{m} v_{k}\left(t, H_{k}^{*}\right) \psi_{k}(\mathbf{a}), \\
P^{*}(t, \mathbf{a}) & =P^{*}(0, \mathbf{a}) K_{t}^{*}(\mathbf{a}) / \Phi(1 ; \mathbf{q}(t), \mathbf{s}(t)) .
\end{aligned}
$$

Then $d l^{*}(t, \mathbf{a}) / d t=l(0, \mathbf{a}) K_{t}^{*}(\mathbf{a}) F_{t}^{*}(\mathbf{a})=l^{*}(t, \mathbf{a}) F_{t}^{*}(\mathbf{a})$. Next,

$$
\begin{aligned}
\int_{A} l^{*}(t, \mathbf{a}) d \mathbf{a} & =\int_{A} l(0, \mathbf{a}) K_{t}^{*}(\mathbf{a}) d \mathbf{a}=N(0) \Phi(1 ; \mathbf{q}(t), \mathbf{s}(t))=N^{*}(t) ; \\
\int_{A} g(\mathbf{a}) l^{*}(t, \mathbf{a}) d \mathbf{a} & =\int_{A} g(\mathbf{a}) K_{t}^{*}(\mathbf{a}) l(0, \mathbf{a}) d \mathbf{a}=N(0) \Phi(g ; \mathbf{q}(t), \mathbf{s}(t))=G^{*}(t) ; \\
\int_{A} h(\mathbf{a}) P^{*}(t, \mathbf{a}) d \mathbf{a} & =\int_{A} h(\mathbf{a}) K_{t}^{*}(\mathbf{a}) P(0, \mathbf{a}) / \Phi(1 ; \mathbf{q}(t), \mathbf{s}(t)) d \mathbf{a} \\
& =\Phi(h ; \mathbf{q}(t), \mathbf{s}(t)) / \Phi(1 ; \mathbf{q}(t), \mathbf{s}(t))=H^{*}(t) .
\end{aligned}
$$

It means that functions $l^{*}(t, \mathbf{a}), N^{*}(t), G_{i}^{*}(t), H_{k}^{*}(t)$ satisfy system (3.5)-(3.6).

Conversely, let $l(t, \mathbf{a}), N(t), G_{i}(t), H_{k}(t)$ solve system (3.3) for $t \in[0, T)$. For now, define the functions $q_{i}^{*}(t), s_{k}^{*}(t), i=1, \ldots, n, k=1, \ldots, m$ by relations:

$$
\begin{gathered}
q_{i}^{*}(t)=\int_{0}^{t} u_{i}\left(x, G_{i}(x)\right) d x, \text { and } s_{k}^{*}(t)=\int_{0}^{t} v_{k}\left(x, H_{k}(x)\right) d x ; \text { then } \\
d l(t, \mathbf{a}) / l(t, \mathbf{a})=\sum_{i=1}^{n} \varphi_{i}(\mathbf{a}) d q_{i}^{*}(t)+\sum_{k=1}^{m} \psi_{k}(\mathbf{a}) d s_{k}^{*}(t), \quad \text { and } \\
l(t, \mathbf{a})=l(0, \mathbf{a}) \exp \left(\sum_{i=1}^{n} \varphi_{i}(\mathbf{a}) d q_{i}^{*}(t)+\sum_{k=1}^{m} \psi_{k}(\mathbf{a}) d s_{k}^{*}(t)\right) \text { for all } t \in[0, T) .
\end{gathered}
$$


Hence, $N(t)=\int_{A} l(t, \mathbf{a}) d \mathbf{a}=\int_{A} l(0, \mathbf{a}) \exp \left(\sum_{i=1}^{n} \varphi_{i}(\mathbf{a}) q_{i}^{*}(t)+\sum_{k=1}^{m} \psi_{k}(\mathbf{a}) s_{k}^{*}(t)\right)$ $d \mathbf{a}=N(0) \Phi\left(1 ; \mathbf{q}^{*}(t), \mathbf{s}^{*}(t)\right)$;

$$
\begin{aligned}
G(t) & =\int_{A} g(\mathbf{a}) l(t, \mathbf{a}) d \mathbf{a} \\
& =\int_{A} g(\mathbf{a}) \exp \left(\sum_{i=1}^{n} \varphi_{i}(\mathbf{a}) q_{i}^{*}(t)+\sum_{k=1}^{m} \psi_{k}(\mathbf{a}) s_{k}^{*}(t)\right) l(0, \mathbf{a}) d \mathbf{a} \\
& =N(0) \Phi\left(g ; \mathbf{q}^{*}(t), \mathbf{s}^{*}(t)\right) ; \\
H(t) & =\int_{A} h(\mathbf{a}) l(t, \mathbf{a}) d \mathbf{a} / N(t)=N(0) M\left(h ; \mathbf{q}^{*}(t), \mathbf{s}^{*}(t)\right) / N(t) \\
& =\Phi\left(h ; \mathbf{q}^{*}(t), \mathbf{s}^{*}(t)\right) / \Phi\left(1 ; \mathbf{q}^{*}(t), \mathbf{s}^{*}(t)\right) .
\end{aligned}
$$

From the definition, $\left\{\mathbf{q}^{*}(t), \mathbf{s}^{*}(t)\right\}$ is the solution of Cauchy problem (3.5)-(3.6) for $t \in[0, T)$. Theorem is proven.

\section{References}

Ackleh AS, Marshall DF, Heatherly HE, Fitzpatrick BG (1999) Survival of the fittest in a generalized logistic model. Math Models Methods Appl Sci 9:1379-1391

Ackleh AS, Fitzpatrick BG, Thieme HR (2005) Rate distribution and survival of the fittest: a formulation on the space of measures. Discret Contin Dyn Syst Ser B 5(4):917-928

Barton MN, Turelli M (1987) Adaptive landscapes, genetic distance and the evolution of quantitative characters. Genet Res 49:157-173

Fisher RA (1999) The genetical theory of natural selection: a complete variorum edition. Oxford University Press, Oxford

Frank SA (1997) The Price equation, Fishers fundamental theorem, kin selection and causal analysis. Evolution 1712:51

Gause GF (1934) The struggle for existence. Williams and Wilkins, Baltimore. http://www.ggause.com/ Contgau.htm

Gorban AN (1984) Equilibrium encircling. Equations of chemical kinetics and their thermodynamic analysis, Nauka, Novosibirsk [in Russian]

Gorban AN (2007) Selection theorem for systems with inheritance. Math Model Nat Phenom 2(4):1-45

Gorban AN, Khlebopros RG (1988) Demon of Darwin: Idea of optimality and natural selection, Nauka (FizMatGiz), Moscow, [in Russian]

Grafen A (2006) A theory of Fisher's reproductive value. J Math Biol 53:15-60

Goodnight KF (1992) The effect of stochastic variation on kin selection in a budding-viscous population. Am Nat 140:1028-1040

Haldane JBS (1990) The causes of evolution, Princeton Science Library. University Press, Princeton

Hofbauer J, Sigmund K (1998) Evolutionary games and population dynamics. Cambridge University Press, London

Karev GP (2000) The effects of non-uniformity in population models. Doklady Math 62:141-144

Karev GP (2003) Inhomogeneous models of tree stand self-thinning. Ecol Model 160:23-37

Karev GP (2005a) Dynamic theory of non-uniform population and global demography models. J Biol Syst 13:83-104

Karev GP (2005b) Dynamics of heterogeneous populations and communities and evolution of distributions. Discret. Continuous Dyn Syst Suppl Vol 487-496

Karev GP (2008) Inhomogeneous maps and mathematical theory of selection. JDEA 14:31-58

Karev G, Novozhilov A, Koonin E (2006) Mathematical modeling of tumor therapy with oncolytic viruses: effects of parametric heterogeneity on cell dynamics. Biology Direct 3 Oct 2006, 1:30 
Kotz S, Balakrishnan N, Jonson N (2000) Continuous multivariate distributions, vol 1, no 2. Wiley, New York

Lande R, Arnold SJ (1983) The measurement of selection on correlated characters. Evolution 37:1210-1226

Lewontin RC (1974) The genetic basis of evolutionary change. Columbia University Press, NY

Li CC (1967) Fundamental theorem of natural selection. Nat Lond 214:504

Liebig J (1876) Chemistry applications to farming and physiology

Nikolaou M, Tam VH (2005) A new modeling approach to the effect of antimicrobial agents on heterogeneous microbial populations. J Math Biol 52:154-182

Novozhilov AS On the spread of epidemics in a closed heterogeneous population. Math Biosci (2009). doi:10.1016/j.mbs.2008.07.010

Page KM, Nowak MA (2002) Unifying evolutionary dynamics. J Theor Biol 219:93

Perthame B (2007) Transport equations in biology. Birkhäuser, Basel

Poletaev IA (1966) On mathematical models of elementary processes in boigeocoenosis, problems of Cibernetics, No 16, pp 171-190 [in Russian]

Price GR (1955) The nature of selection. J Theor Biol 175:389

Price GR (1970) Selection and covariance. Nature 227:520

Price GR (1972) Extension of covariance selection mathematics. Ann Hum Genet 35:484

Rice SH (2006) Evolutionary theory. Sinauer Associates Inc., Sunderland, MA

Robertson A (1968) The spectrum of generic variation. In: Lewontin RC (ed) Population biology and evolution. University Press, Syracuse, p 4

Schuster P, Sigmund K (1983) Replicator dynamics. J Theor Biol 100:533-538

Semevsky FN, Semenov SM (1982) Mathematical modeling of ecological processes, Gidrometeoizdat, Leningrad [in Russian]

Thieme HR (2003) Mathematics in population biology. Princeton University Press, Prinston

Wilson DS, Dugatkin LA (1997) Group selection and assortative interactions. Am Nat 149:336-351

Zeldovich KB, Chen P, Shakhnovich BE, Shakhnovich EI (2007) A first-principles model of early evolution: emergence of gene families, species, and preferred protein folds. PLoS Comput Biol 3(7) 